\title{
LA DIMENSIÓN INTERACCIONAL EN LA SUPERVISIÓN DE PRÁCTICAS DOCENTES. UNA APROXIMACIÓN DESDE LA TRANSDISCIPLINARIEDAD
}

\section{Paola Andreucci ${ }^{1}$}

\section{RESUMEN}

Este trabajo se sostiene en una propuesta de articulación transdisciplinaria sobre la instancia y el proceso de supervisión de profesores desde una óptica interaccional. Se aborda la complejidad epistemológica (Morin, 2007) de la supervisión recurriendo a aportes discursivos de diversas disciplinas: la pedagogía, la filosofía y el lenguaje dialógico. De las primeras se incorporan los principios y reglas de clasificación y enmarcamiento (Bernstein, 1975, 1985, 1999) para precisar y delimitar las características del dispositivo y discurso pedagógico. De la filosofía y de las ciencias dialógicas (Bajtín, 2003, 2008) se releva la generación de subjetividad a partir de una reacción frente al otro y los otros. Se ejemplifica la propuesta con un material de supervisión, ofreciéndose algunas directrices para su aplicación.

Palabras clave: relación supervisor-supervisado, complejidad, discurso pedagógico, discurso dialógico

\section{INTERACTION DIMENSION IN THE SUPERVISION OF TEACHING PRACTICES: A CROSS-DISCIPLINE APPROACH}

\begin{abstract}
This work is based on a joint proposal of a cross-discipline study and a teacher supervision process from a relational perspective. It addresses the epistemological complexity (Morin, 2007) of using supervision discursive contributions from various disciplines: pedagogy, philosophy and dialogic language. The first of them include principles and rules of classification and framing (Bernstein, 1975, 1985, 1999) to clarify and define the characteristics of the device and pedagogical discourse. From philosophy and science dialogic (Bajtín, 2003, 2008), it relieves the generation of subjectivity from a re-action to others. The proposal is exemplified in a supervisory material offering some guidelines for its implementation.
\end{abstract}

Keywords: supervisor-supervisee relationship, complexity, teaching speech, dialogic speech

1 Académica e investigadora. Facultad de Educación, Universidad Diego Portales, Santiago, Chile. Contacto: pandreucci@manquehue.net 


\section{LA DIMENSIÓN INTERACCIONAL EN LA SUPERVISIÓN DE PRÁCTICAS DOCENTES. UNA APROXIMACIÓN DESDE LA TRANSDISCIPLINARIEDAD}

\section{Introducción}

Tradicionalmente la "super-visión" instala la presencia de una mirada jerárquica y oficial que emula al discurso vertical propuesto por Bernstein (1999) y que tiene la capacidad de proyectar la visión sobre lo manifiestamente develado.

Supone la presencia de un profesional experto, un supuesto saber omnisciente y omnipresente, capaz de ponderar el curso de las intervenciones en el aula y evaluar la pericia y el desempeño desplegados por quienes están a cargo de él en calidad de supervisados. Puede y suele operar como un mecanismo de control que examina "desde arriba" lo que el docente en formación hace y/o dice que hace en el aula con sus estudiantes.

Las escuelas de formación de profesores han ido adoptando una actitud de decidido apoyo a la práctica de supervisión. En esta dirección se han diseñado estrategias de monitoreo y evaluación de los desempeños docentes que, incluso apoyados en técnicas de observación de videos de clases realizadas y/o de muestras de desempeño en el aula, consideran focalmente la implementación de habilidades y destrezas, actitudes y valores que deben ser manifestados por el profesor en formación.

Estas iniciativas acompañadas de instancias de autoevaluación de lo/as docentes en formación a lo largo del proceso de práctica, posibilitan que el/la supervisor/a pueda estar atento/a a las distintas dimensiones del formar y orientar, y el formando pueda reflexionar periódicamente sobre sus prácticas (Schön, 1992, 1998). Un profesional que reflexiona sobre su quehacer se hace consciente de sus dificultades y logros y está en condiciones de corregirlos y/o 
mejorar su desempeño, siguiendo el argumento central de Schön. No obstante, esta reflexión requiere ser acompañada de la visión del profesional experto que identifica, focaliza, retroalimenta y visibiliza con claridad las formas de abordaje correctivo a lo evaluado en déficit y potencia lo evaluado como competente.

El valor de transmisión efectiva del dispositivo de supervisión -desde profesores seniors a novatos- puede cuestionarse, no obstante, por al menos dos poderosos argumentos: estimar el grado de corrección/modificación que adopta el curso de las prácticas docentes luego de las instancias de supervisión. Es relativamente reconocido que el profesor en formación opera desde sus teorías implícitas o creencias, que presentan tanto estabilidad y cambio -como señalan Nettle (1998) y Latorre, Pérez-García y Blanco-Encomienda (2009)_ y desde sus propias significaciones y/o de sus recuerdos referentes a cómo él fue formado en la sala de clases, más que desde las prácticas adecuadamente fundamentadas teórica y metodológicamente para la generación de diversos tipos de aprendizajes. El segundo argumento apunta a cuestionar en qué medida las intervenciones sugeridas por un profesor-supervisor que no está presencialmente en el aula -o que lo está pero en otro rol- pueden considerarse las más adecuadas para una particular situación de aprendizaje en un contexto interaccional determinado.

Por otra parte, el cuestionamiento se incrementa cuando se deja de lado lo que se desarrolla en la trama relacional en que la transmisión de habilidades, actitudes y valores ocurre: la práctica de supervisión. Incluso se llega a invisibilizar esta instancia y proceso interaccional considerándolo un medio para el logro de otra cosa, más que un proceso formativo generador de aprendizajes en sí mismo. Pareciera que se habla de lo que ocurre allá afuera -en la práctica de los profesores en formación con sus estudiantes- desligándolo de lo que ocurre acá adentro -en la práctica de supervisión entre el formador y su/s formando/s-. Esto ocurre a pesar de que la literatura ha reparado, en este último tiempo y quizás muy recientemente, sobre la importancia del desarrollo de las habilidades interpersonales en el espacio de supervisión (Rippon y Martin, 2006; Cid, Pérez y Sarmiento, 2011). 
Si se desplaza la concepción relativa al poder/control del supervisor -aunque no se prescinda totalmente de ella, ya que en términos de Bernstein (1975) es necesario que operen reglas de distribución, de recontextualización y evaluativas en cualquier dispositivo pedagógico- y se permite una mirada a la complejidad interaccional del fenómeno, es posible detectar en la instancia de supervisión la presencia de diversas dimensiones: a) una dimensión formativa o pedagógica, b) una dimensión orientadora o estratégica y, c) una dimensión evaluativa de la gestión institucional.

La instancia procesal de supervisión supone, por tanto, el desarrollo e implementación de competencias orientadas a generar paulatina autonomía, propiciando una postura personal y profesional en el profesor que se inicia en su recorrido como docente, interrogando críticamente su docencia y el sistema en que se inserta pero, a la vez, ajustándose a los procedimientos institucionales vigentes.

Adicionalmente, cuando se producen eventos críticos y/o situaciones de especial dificultad de enfrentamiento con lo/as estudiantes en el aula, se propondría atender a lo que, apoyándose en el modelo clínico, se ha denominado "procesos paralelos" -lo que ocurre con un/a estudiante se replica en la instancia de supervisiónevitando así la "doble traumatización", es decir, el proceso en el cual el profesor tiene relaciones estresantes en el trabajo y simultáneamente experimenta conflictos con su supervisor, requiriendo especial atención para retomar efectivamente su labor y recuperar su motivación por el trabajo (Orlinsky y Ronnestad, 2005; Ladany, Friedlander y Nelson, 2005 ).

\section{Aproximación transdisciplinaria: epistemología de la complejidad}

Desde la epistemología de la complejidad, siguiendo inicialmente a Morin (2007a) y otros investigadores que han relevado esta visión sobre los procesos educativos y la educación en general (Couto y Vásquez, 2011; Flores, 2008; Motta, 2002; Oliva, 2010, Zabala y Vidiella, 1999), se levanta una alerta sobre los peligros de la simplificación y naturalización de las disciplinas y el apoyo en datos 
empíricos sin fundamentación conceptual pertinente. En palabras del autor: "Es necesario desarrollar la aptitud (...) para ubicar todas sus informaciones en un contexto y en un conjunto. Es necesario enseñar los métodos que permiten aprehender las relaciones mutuas y las influencias recíprocas entre las partes y el todo en un mundo complejo" (Morin, 2007b: 15). Comprender lo humano y los procesos de formación es comprender su unidad en la diversidad, su diversidad en la unidad. Se hace necesario comprender la unidad educativa en lo múltiple, la multiplicidad de enfoques educativos en lo uno y al unísono.

Por otra parte, la generación de dispositivos de supervisión pedagógica centrados exclusiva o fundamentalmente en los contenidos disciplinares, en la planificación de la clase y en el desarrollo de los sistemas evaluativos a implementar, tiende a despersonalizar la labor del profesor en formación y de su supervisor, insertos en un enfoque interaccional de la enseñanza: no se enseña a través de un computador o de la mera implementación de diversas didácticas, aunque se apoye el profesor en estos medios o en otros para complementar sus discursos pedagógicos sobre el qué y cómo aprenden sus estudiantes. El discurso interaccional del profesor es relevante para que el acto educativo ocurra.

En opinión de la autora de este trabajo (Andreucci, 2008), se justifica por la naturaleza del objeto de estudio aproximarse al mismo de un modo complejo, apelando a dimensiones tanto pedagógicas como filosóficas y discursivas, dadas las acciones comprendidas en la generación, mantención y desarrollo del dispositivo de supervisión. "La supremacía de un conocimiento fragmentado según las disciplinas impide a menudo operar el vínculo entre las partes y las totalidades y debe dar paso a un modo de conocimiento capaz de aprehender los objetos en sus contextos, sus complejidades, sus conjuntos" (Morin, 2007b, p. 15).

Se concibe la supervisión pedagógica como un espacio privilegiado para la aplicación de la pluri y transdisciplinariedad, lo que siguiendo a Nicolescu es descrito como: "(...) el estudio del objeto de una sola y misma disciplina por medio de varias a la vez. El objeto 
saldrá así enriquecido por la convergencia de varias disciplinas. El conocimiento del objeto dentro de su propia disciplina se profundiza con la aportación pluridisciplinaria fecunda" (Nicolescu, 1998: 2) Es decir, el objeto de estudio es construido relacionalmente por la perspectiva no neutra ni ingenua del investigador. Se intencionan las relaciones y la interpretación o traducción del fenómeno observado u objeto, no se trata de una simple enunciación de contenidos sino que se decide instalar el conocimiento en relación y en la perspectiva del "observador". Además, la relación no es simplemente binaria o no debiera reducirse a ello, en una concepción pluridisciplinaria. La experiencia es siempre relacional, presenta diversos puntos de vista, por ello es irreductible y a la vez compartible en la trama interactiva. Metafórica o análogamente, "la moneda no solamente tiene dos caras, tiene múltiples caras". Lo transdisciplinario, por su parte, instala una reforma paradigmática y no meramente programática para la educación. Se releva la noción de "campo educacional" más que de una disciplina educativa específica y reductible. En este campo, la pregunta fundamental para la educación tiene que ver con la aptitud, actitud y decisión como "formadores", para organizar el conocimiento de un nuevo "modo": como "tejido". Interpretando "la realidad" como un entrelazado de eventos, acciones, retroacciones, determinaciones, azares y contradicciones en la perspectiva de la incertidumbre y del desafío interactivo. Si bien lo "pluri" refiere a cantidad o número de puntos de vista, lo "trans" alude a relaciones recíprocas, actividades de cooperación, interdependencia, intercambio e interpenetración (Motta, 2002). Lo trans supera a lo pluri, no obstante ambas dimensiones o aproximaciones -lo pluri y lo transdisciplinario- en su conjunto, permiten la emergencia de un nuevo fenómeno en la trama interactiva.

La capacidad de "formación en" y "transmisión interactiva" de este entramado se constituye en un elemento central para trabajar como supervisor y capacitar a profesores. Es esencial aclarar y entender, no obstante, cómo un supervisado se aproxima y experimenta la situación, realiza un análisis o participa en la supervisión y presentación del caso problema. Reflexionar sobre lo que el formando percibe, cómo se relaciona con ello y qué reacciones expresa es clave para el proceso de aprendizaje así como para su evaluación. 
Resulta esencial que los argumentos utilizados sean transparentes y estén presentados de manera que sea posible que otros puedan evaluar la forma en que se percibieron, cómo se llegó a las conclusiones y tener una oportunidad de ofrecer interpretaciones alternativas. Este planteamiento, sostenido por Schön $(1992,1998)$ y, en algún sentido, por Perrenoud (2004) en su argumentación sobre la práctica reflexiva, es similar al ofrecido por Bourdieu (2007) en su énfasis en la pedagogía racional y, más específicamente, por Bernstein (1985) en pedagogías visibles y su atención a los riesgos de la invisibilidad de los procesos para la educación.

\subsection{Discurso pedagógico}

Considerando el discurso pedagógico como un dispositivo esencialmente, pero no meramente, lingüístico, Bernstein y Díaz (1985) enfatizan la producción discursiva en su dimensión de mecanismos de poder y de control simbólico para las posiciones o posicionamientos y reposicionamientos de sujetos dentro de órdenes específicos. Por lo tanto, el régimen de su producción discursiva involucra agentes sociales y discursos, y la relación posicional entre los mismos en contextos social e históricamente determinados.

Desde este punto de vista, el discurso pedagógico opera y puede considerarse como un dispositivo de reproducción de formas de conciencia específica a través de la producción de reglas específicas que regulan relaciones sociales específicas, entre categorías específicas tales como la de transmisores y adquirentes, formadores y formandos, supervisores y supervisados. A esto se alude con los así denominados mecanismos de poder y de control simbólicos, en contraposición a mecanismos imaginarios o reales en tanto su valor de verdad absoluta, naturalista o esencialista.

Según Bernstein (1975), desde su distinción entre pedagogías visibles e invisibles, existen tres reglas de regulación de la relación entre transmisores y adquirentes, a saber: reglas de jerarquía, reglas de secuencia y los criterios. Estas reglas constituyen los rasgos básicos de cualquier relación de transmisión y adquisición, y por lo tanto, son rasgos cruciales para la educación en tanto proceso formativo. 
Se considera, además, que la forma que toma la relación jerárquical vertical afecta tanto las reglas de secuencia como los criterios.

En lo específico, las reglas de jerarquía regulan lo que debe ser un transmisor y lo que debe ser un adquirente o supervisado en este caso. Se espera que el aprendiz o formando aprenda a ser un tipo particular de aprendiz y que el transmisor aprenda a ser un tipo particular de transmisor. Estas reglas determinan la forma jerárquica de la transmisión. Ellas establecen sus reglas de conducta. Las reglas de secuencia, por su parte, regulan la temporalidad; cualquiera transmisión se extiende en el tiempo. Como consecuencia, algo ocurre antes y algo viene después. Finalmente, los criterios en tanto transferencia de esquemas de transmisión/adquisición vinculan, necesariamente, la transferencia de referentes con los cuales se espera que el aprendiz controle, explore y evalúe su propia conducta -en un sentido amplio- y la de los otros. Se trataría de una dimensión de introspección y de meta reflexión.

Desde la perspectiva del discurso vertical y horizontal, Bernstein (1999) va a aportar una distinción aún más clara. El discurso vertical es fundamentalmente de enmarcamiento fuerte, seleccionado, secuenciado, de ritmo intenso y evaluado formalmente. Este enmarcamiento puede ser explícito, como ocurre en la pedagogía tradicional, o implícito, como ocurre en la denominada pedagogía progresista. El discurso horizontal -habla espontánea en el aula- y narrativas confesionales en antropología, algunas formas de psicología y sociología, posibilitan relaciones interpersonales más "penetrativas", cercando la intimidad e interpelando la subjetividad.

La estructuración de las relaciones sociales genera las formas del discurso, no obstante, el discurso a su vez estructura una forma de conciencia, su orientación de sentido y realización, y motiva formas de intercambio interpersonal en contextos simbólicos o culturales determinados, como el de supervisión, en este caso.

\subsection{Discurso dialógico}

La dirección del discurso reviste especial interés cuando se aborda la complejidad relacional del dispositivo de supervisión. ¿Hacia quiénes 
se habla o hacia dónde se dirige el discurso? y ¿desde qué lugar se habla?

El lugar desde el cual se habla ha sido profundamente revisado y revisitado por Mijaíl Bajtín $(2003,2008)$, quien refiriéndose a la relación entre autor y personaje o héroe en la actividad artística, señala "la pluralidad de voces y conciencias independientes e inconfundibles, la auténtica polifonía de voces autónomas, viene a ser, en efecto, la característica principal de la novelas de Dostoievski" (Bajtín, 2003, p. 15). Refiriéndose a la distinción-indistinción, sujeto-objeto en la relación con los otros, aporta con la siguiente afirmación: "los héroes principales de Dostoievski, efectivamente, son, según la misma intención artística del autor, no sólo objetos de su discurso, sino sujetos de dicho discurso con significado directo" (Bajtín, 2008a, p. 15).

Siguiendo a Koshinov en el prólogo del texto "Problemas de la poética de Dostoievski" (1979), aclara que "de esta manera, en el universo artístico de Dostoievski, el autor narrador o participante de la obra es el que puede equipararse a los personajes (utilizando el término bajtiniano, se podría hablar de la voz del autor) y no el autor real" (Koshinov, 2003, p.10).

El lenguaje es el que permite el encuentro interaccional, dado que no es arbitrario en tanto posee una estructura y un sentido que le subyace. Es a esta estructura -formada por un sistema de signos-y a este sentido -intencionalidad discursiva- a la que se puede acceder mediante la interpretación del discurso (Ortí, 1994).

Entonces, es mediante el análisis e interpretación del discurso que se hace posible acceder a las significaciones. Esto, ya que "los discursos entrañan, (...) la existencia de las significaciones culturales de la comunicación simbólica, estructurada por un sistema de signos intersubjetivos o lenguaje, y atravesada por el sentido subjetivo (consciente o no consciente) del actor hablante" (Ortí, 1994, p. 190).

\section{Ejemplificación ilustrativa}

La estudiante Yasna tiene 20 años y cursa tercero medioadministración; ha repetido dos veces. Vive con su madre, abuela 
materna y hermano de ocho años. Presenta escasa comunicación con su madre, en ocasiones "nos peleamos y nos decimos cosas fuertes". Con su abuela y hermano dice llevarse "muy bien". Tiene una prima que ella siente "como hermana", señala: "es como la prima-hermanaamiga que nunca tuve".

Ha presentado problemas de conducta en los colegios a los que ha asistido. Le incomodan las normas y los protocolos. Entra en conflicto cuando defiende a sus compañeras. Al respecto, señala: "soy súper jugá por mis amigas...yo ya soy como soy, no voy a cambiar a esta edad, porque no quiero...soy mala pá defenderme yo de la gente, pero defiendo a todas las personas que quiero con uñas y dientes".

Presenta un rendimiento "bueno". Su actual promedio de calificaciones es de 5,02. Le gustaría, a futuro, "poder estudiar medicina forense porque veo series y es re' choro". Tiene decidido hacer el servicio militar, una vez que termine la enseñanza media.

Solo a veces siente ganas de compartir, prefiere "proteger" a sus compañeras. Declara: "siempre hay que proteger al más débil aunque él no se dé cuenta."

En relación a su prima señala: "con ella nos entendemos, consumimos (pasta base) y nos olvidamos del mundo...no hay na' que valga la pena de ser vivido...no tengo ningún futuro... ¿y Ud. qué piensa? (refiriéndose a la profesora)"

Esta conversación entre estudiante y profesora, como otras sostenidas con anterioridad, se producen entre clases al salir a recreo.

La profesora en formación, de 22 años de edad, comenta en supervisión: "no puedo entender lo que le ocurre a esta alumna... me agrede directamente en una clase y, en el pasillo me pide consejo, siendo súper dulce...como que invierte la situación... ¡no sé qué hacer y cómo responderle a sus preguntas... entremezcla lo personal

2 En la escala de 1 a 7. 
con su situación como estudiante!... ¿Ud. qué piensa? (refiriéndose a la supervisora)".

La supervisora pregunta: "ipuedes ejemplificar ambas posiciones?, no logro comprender del todo a qué te refieres con una u otra posición de tu alumna y cómo tú reaccionas al respecto".

La profesora se queda en silencio y esboza una expresión de asombro que luego se torna en molestia. Verbaliza: “icómo no entiende, de eso hemos estado hablando estas dos últimas supervisiones!"

La supervisora intenta calmar infructuosamente a su supervisada con afirmaciones como ésta: "se trata de una nueva situación y debemos revisar lo particular que ella presenta, lo que se repite de otras clases, de los antecedentes personales que te ha relatado con su situación actual como estudiante". Agrega: "el ejercicio de la docencia consiste en analizar esto, no sólo el contenido disciplinar y la programación de la clase, sino también la relación establecida con tus estudiantes, en especial, cuando son tan cercanas en edad a ti".

Ante el señalamiento de la supervisora, la profesora replica: "ino necesito que me calme, yo puedo sola... lo que necesito es que me enseñe, aunque sea desde su particular postura pedagógica!" Luego de esto se retira de la sesión de supervisión señalando: "voy a solicitar traslado de supervisión de práctica, Ud. y yo no nos entendemos. Gracias por el esfuerzo de todos modos".

La profesora en formación se ausenta de su práctica y presenta licencia médica por "estrés".

En primer lugar, es posible entender la instancia de supervisión de prácticas docentes como un género discursivo en sí mismo (Bajtín, 2008b). Un género, no obstante, en el cual las formas de los discursos, es decir, los principios internos de su construcción o develamiento y sus fundamentos sociales, fueron dados por sentados y no se han sometido a ningún tipo de análisis aún (Bernstein, 1999). En la aproximación a la trama discursiva 
ejemplificada, el punto de inicio está dado por distinguir las dos formas fundamentales del discurso -vertical y horizontal- que han sido contrastados: el discurso jerárquico o de supuesto saber del supervisor y el discurso más intimista o de búsqueda de empatía y complicidad por parte del supervisado. El segundo, al no recibir respuesta -o reacción esperada en este registro- retorna a una lógica escolarizada interpelando el saber "disciplinario" de su supervisora, de un modo que genera inicialmente un distanciamiento afectivo-cognitivo entre ambas y luego un distanciamiento en la realidad: en el acto de abandono de la instancia de supervisión. La supervisora no logra atender a la complementariedad de ambas lógicas discursivas y se ampara en su autoridad. Su repertorio estaría limitado en estrategias y en potencial analógico, ya que es preciso observar cómo desde la relación de la docente con su alumna se produce un "proceso paralelo" o analógico de esta profesora con su supervisora. El incidente ocurrido con la estudiante se reedita en el espacio de supervisión y varias voces, como lo propone la polifonía bajtiniana -estudiante, profesora, supervisora- quedan atrapadas en una maraña comunicacional donde el discurso pierde su explícita intencionalidad y trascendencia. ¿Cuál es el autor y cuál su héroe? Algo de lo oracular es demandado implícitamente a la supervisora, quien sería incapaz de anticipar el devenir discursivo y el enactment o pasaje al acto de su adquiriente. Los lugares o posiciones ocupados por la supervisora para su supervisada -sabia, madre, amiga, compañera de ruta, colega- se encontraban ocultos en las claras reglas de jerarquía y recontextualización que a priori fueron definidas para el espacio de supervisión. Sin embargo, las funciones y posiciones relacionales -frame relationships-al interior del proceso resultaron indeterminadas hasta el momento del "incidente", ya que su temporalidad y efectividad en la transmisión de los mensajes no se había puesto a prueba hasta este instante.

Los procedimientos de estratificación generan reglas de distribución que controlan el flujo que va desde el reservorio social hacia el repertorio individual, aportando un acumulado de creencias respecto a lo que se es o el lugar simbólico que se ocupa en un determinado género discursivo, supervisora o supervisada, en este caso. Esta perspectiva representacional, no obstante, limita la 
mirada sobre el tejido relacional de carácter recursivo, dialógico y dialéctico que opera en la escena de supervisión. Bajo una invitación de mutualidad se despliega el artesanado de la labor formativa, la cual puede llegar a violentar al sujeto en formación, en el peor de los casos, o desafiarlo sin intimidarlo, aportando a su generación como sujeto profesional con una postura personal frente a su quehacer, en el mejor escenario.

En este caso, la "violentación" relacional opera también como provocación discursiva y apuesta por la no vulneración del otro, sino que por el desafío a su constitución subjetiva. La supervisora no revela los pensamientos y sentimientos que le guían en su desplazamiento desde una fase indagativa a otra. Ella "reflexiona desde la acción, pero no reflexiona sobre su reflexión desde la acción” (Schön, 1998, p.120). La supervisada podría descubrir cómo su relación con la supervisora se parece a la relación de su alumna con ella como su docente, especialmente en la ambivalencia entre la demanda "voraz" y la búsqueda de control. Del mismo modo que su alumna, la supervisada se siente bloqueada en la relación de ayuda, deseando más de lo que se le entrega o de lo que percibe estaría recibiendo. Al parecer, esto la impacta emocional y subjetivamente y la hace desistir de la interacción.

Los límites relacionales tendrán que ser redefinidos si ambas partes se proponen, consciente e inconscientemente, trabajar en la dirección de la reparación de esta relación en pro del proceso formativo. La recuperación de sentidos y su valor simbólico supone traducir lo sucedido y develar explícitamente el patrón relacional desplegado en la sesión de supervisión mediante un proceso meta reflexivo. Es labor del par supervisora-supervisada redefinir y reencuadrar el espacio formativo como un dispositivo pedagógico jerarquizado y, a la vez, flexible, donde la supervisora genera a su supervisada; no obstante -en opinión de la autora de este trabajo- la supervisada restituye o no el lugar de supervisora a la formadora experimentada y recobra o reenfoca la motivación por su trabajo. Siguiendo a Schön (1998), se hace necesario, además, ampliar los límites de la reflexión para incluir el sentido o significados de la demostración argumentativa del supervisor, reflexionando en su propia reflexión desde la acción y, de 
este modo, la supervisada podría tener acceso a las fuentes discursivas de la actuación de su supervisora.

Evitar la "doble traumatización" (Orlinsky y Ronnestad, 2005) resulta prioritario, ya que los síntomas son evidentes y es en el escenario interaccional donde es posible evitar, reparar el daño y propiciar nuevas alternativas de enfrentamiento y definición subjetiva.

\section{Conclusiones y comentarios finales}

El enfoque utilizado en el presente trabajo no repara en las temáticas o contenidos disciplinares de la instancia de supervisión, sino que pone el foco en la dimensión relacional, en el framing, en el encuentro dialógico entre supervisor y supervisado como una unidad de análisis en sí misma que requiere una permanente reflexión procesal, introspección y atención a los denominados procesos paralelos, como intentó reflejar la ejemplificación ilustrativa.

Dicho de otro modo, la pregunta, y las respuestas tentativas no son por el "qué" y tampoco de modo central por el "cómo", sino por los autores y protagonistas del proceso de supervisión, en tanto dispositivo y en tanto discurso simbólico o pleno de significados para unos y otros.

Se plantea, por tanto, que el encuentro discursivo logra más conocimiento sobre el otro y la situación que cualquiera otra práctica que refiera sentidos o significados a develar y construir. Más específicamente, no re-presenta los sentidos, sino que los presenta o genera.

El sujeto y la subjetividad se producirían en el encuentro dialógico que define la posición de ambos -supervisor y supervisadoy la modifica en función de la sensibilidad a las claves discursivas generadas entre ellos. El sujeto, su reacción, su postura frente al otro, por lo tanto, es producido en el encuentro y el supervisor posibilita que ello ocurra. 
La consideración de lo inconsciente, no explícito, a juicio de la autora del presente trabajo, le imprime una necesaria "ambigüedad" al trabajo de supervisión, posibilitando que se circule de enmarcamientos fuertes a débiles (Bernstein, 1985) con inusitada flexibilidad y fluidez.

Al igual que en la relación profesor-alumno, el encuentro dialógico entre supervisor y supervisado -mediado por el "problema o situación" a supervisar-adquiere su carácter propio, fundamental, y se construye en la adecuación permanente del supervisado de hablar no sólo "para" el otro (supervisor), sino "por" otro (figuras significativas: estudiante, par, amiga, hija). El supervisor contribuiría con su mutualidad, es decir se tendería a hablar de "mutua adecuación" (Arfuch, 2010).

La complejidad relacional entre supervisor y supervisado constituye el componente procesal principal para abordar una evaluación comprensiva de esta instancia formativa. La acción de la supervisada -en la ejemplificación ilustrativa-constituye una reacción a una acción anterior, que ella misma genera y trae a colación en su discurso; "icómo no entiende, de eso hemos estado hablando todas estas sesiones!". No sólo su acción anterior influye en su reacción, sino también la anticipación de su respuesta, fijando su postura frente al otro y generando sentidos.

Esto último constituiría la esencia de lo dialógico y lo fundamental del proceso de supervisión desde una perspectiva relacional que repara en los actores del proceso formativo. El desafío es incorporar esta importante dimensión del "quién" al "qué" -aspectos disciplinares- y al "cómo" -aspectos didácticos- de la supervisión, sin olvidar la complejidad del fenómeno (Morin, 2007; Motta, 2002; Oliva, 2010) ni subestimar el valor preventivo del aprendizaje interaccional para la práctica docente diaria.

\section{Referencias bibliográficas}

Andreucci, P. (2008). De la transferencia del analista a la transferencia del maestro. Un desafio transdisciplinario. Tesis de magíster no publicada. 
Universidad Metropolitana de Ciencias de la Educación. Santiago de Chile.

Arfuch, L. (2010). La Entrevista, una invención dialógica. Buenos Aires: Editorial Paidós.

Bajtín, M. (2008a). Autor y personaje en la actividad estética en Estética de la creación verbal ( $2^{\mathrm{a}}$ ed.) Buenos Aires: Editores Siglo Veintiuno. (T. Bubnova, traductora) pp. 13-190.

Bajtín, M. (2008b). El problema de los géneros discursivos en Estética de la creación verbal ( $2^{\mathrm{a}}$ ed.) Buenos Aires: Editores Siglo Veintiuno. (T. Bubnova, traductora) pp. 245-287.

Bajtín, M. (2003). Problemas de la poética de Dostoievski (4a ed.) México: Fondo de Cultura Económica. Por Ayax Lafarga.

Bernstein, B. (1975). Visible and Invisible Pedagogies. En Class, Codes and Control, Vol. III, Towards a Theory of Educational Transmissions. London: RKP.

Bernstein, B. y Díaz, M. (1985). Hacia una teoría del discurso pedagógico. Revista Colombiana de Educación, 15, Versión digital sin páginas. Traducido de Collected Original Resources in Education (CORE), Vol. 8, n. ${ }^{\circ}$ 3, 1984.

Bernstein, B. (1999). Vertical and Horizontal discourse: an essay. British Journal of Sociology of Education, 20 (2), pp. 157-173.

Bourdieu, P. (2007). Cosas Dichas. (M. Mizraji, trad.) Barcelona: Editorial Gedisa. Choses Dites, Paris: Les Éditions de Minuit.

Cid, A; Pérez, A. y Sarmiento, J. (2011). La tutoría en el practicum. Revisión de la literatura. Revista Española de Educación 354, enero-abril, pp. 127-154.

Couto, M.D. y Vázquez, N. Complejidad, transdisciplinariedad y educación. Odous Científica. Revista de la Facultad de Odontología de la Universidad de Carabobo [versión electrónica sin páginas] (s.f.). Recuperado el 11 de febrero de 2011, de: http://servicio.cid.uc.edu.ve/odontologia/ revista/v5n2/v-2-3.pdf

Flores-González, L. M. (2008). Posiciones y Orientaciones Epistemológicas del Paradigma de la Complejidad. Cinta Moebio, 33, 195-203. Disponible en: http:// www.moebio.uchile.cl/33/flores.html

Koshinov, V. (2003). Prólogo. En M. Bajtín (autor), Problemas de la Poética de Dostoievski, pp. 3-12. México: Fondo de Cultura Económica.

Ladany, N. Friedlander, M. y Nelson, M. L. (2005). Critical events in 
psychotherapy supervision: An interpersonal approach. Washington: American Psychological Association.

Latorre Medina, M. J.; Pérez-García M. P. y Blanco-Encomienda, F. J. (2009). Análisis de las creencias que sobre la enseñanza práctica poseen los futuros maestros especialistas en educación primaria y en educación física. Un estudio comparado. Revista Electrónica Interuniversitaria de Formación del Profesorado, 12(1), 85-105. Enlace web: http:// www. aufop.com. Consultada en 17/04/2011.

Morin, E. (2007a). Introducción al pensamiento complejo. Edición española a cargo de Marcelo Pakman. Buenos Aires: Editorial Gedisa.

Morin, E. (2007b). Los siete saberes necesarios para la educación del futuro. Elaborado para la UNESCO como contribución a la reflexión internacional sobre cómo educar para un futuro sostenible. Buenos Aires: Ediciones Nueva Visión.

Motta, R. D. (2002). Complejidad, educación y transdisciplinariedad [versión electrónica sin páginas]. Revista Polis Académica. Universidad Bolivariana, 1(3). Disponible en: http://revistapolis.cl/3/motta3.doc.

Nettle, E. B. (1998). Stability and change in the beliefs of student teachers during practice teaching. Teaching and Teachers Education, 14, 193204.

Nicolescu, B. (1998). La transdisciplinariedad, una nueva visión del mundo. Paris: Edit. Du Rocher.

Oliva, I. (2010). Breve cartografía de una disonancia epistémica: educación, complejidad y reforma. Revista Polis Académica. Universidad Bolivariana, 9 (25), 321-335.

Orlinsky, D. y Ronnestad, M. H. (2005). How psychotherapists develop: A study of therapeutic work and professional growth. Washington: American Psychological Association.

Ortí, A. (1994). La apertura y el enfoque cualitativo o estructural: la entrevista abierta semidirectiva y la discusión de grupo. En: Alvira, F.; Ibáñez, J. y García, M. (editores). El análisis de la realidad social: Métodos y Técnicas de investigación. Madrid: Alianza Editorial.

Perrenoud, P. (2004). Desarrollar la práctica reflexiva en el oficio de enseñar. Profesionalización y razón pedagógica. Barcelona: Graó.

Rippon, J.H. y Martin, M. (2006). What makes a good induction supporter? Teaching and Teachers Education, 22(1), pp. 84-99.

Schön, D. (1992). La formación de profesionales reflexivos: hacia un nuevo diseño de la enseñanza y el aprendizaje en las profesiones. Barcelona: Paidós. 
202 LA DIMENSIÓN INTERACCIONAL EN LA SUPERVISIÓN DE PRÁCTICAS DOCENTES. UNA APROXIMACIÓN DESDE LA TRANSDISCIPLINARIEDAD - P. Andreucci

Schön, D. (1998). El profesional reflexivo. Cómo piensan los profesionales cuando actúan. Barcelona: Paidós.

Zabala-Vidiella, A. (1999). Enfoque globalizador y pensamiento complejo: una respuesta para la comprensión e intervención en la realidad. Barcelona: Graó.

Recibido: 01/03/2011

Aceptado: 28/04/2011 